\title{
Human osteoclasts/osteoblasts 3D dynamic co-culture system to study the beneficial effects of glucosamine on bone microenvironment
}

\author{
ELISABETTA LAMBERTINI ${ }^{1}$, LETIZIA PENOLAZZI ${ }^{1}$, ASSUNTA PANDOLFI $^{2}$, DOMITILLA MANDATORI ${ }^{2}$, \\ VINCENZO SOLLAZZO ${ }^{3}$ and ROBERTA PIVA ${ }^{1}$ \\ ${ }^{1}$ Department of Neuroscience and Rehabilitation, University of Ferrara, I-44121 Ferrara; \\ ${ }^{2}$ Department of Medical, Oral and Biotechnological Sciences, Center for Advanced Studies and Technology - CAST, \\ University G. d'Annunzio of Chieti-Pescara, 66100 Chieti; ${ }^{3}$ Centro di Medicina, I-44124 Ferrara, Italy
}

Received October 13, 2020; Accepted November 24, 2021

DOI: $10.3892 /$ ijmm.2021.4890

\begin{abstract}
Glucosamine (GlcN) functions as a building block of the cartilage matrix, and its multifaceted roles in promoting joint health have been extensively investigated. However, the role of GlcN in osteogenesis and bone tissue is poorly understood, mainly due to the lack of adequate experimental models. As a result, the benefit of GlcN application in bone disorders remains controversial. In order to further elucidate the pharmacological relevance and potential therapeutic/nutraceutic efficacy of GlcN, the effect of GlcN treatment was investigated in human primary osteoclasts (hOCs) and osteoblasts (hOBs) that were cultured with two-dimensional (2D) traditional methods or co-cultured in a 3D dynamic system more closely resembling the in vivo bone microenvironment. Under these conditions, osteoclastogenesis was supported by hOBs and sizeable self-assembling aggregates were obtained. The differentiated hOCs were evaluated using tartrate-resistant acid phosphatase assays and osteogenic differentiation was monitored by analyzing mineral matrix deposition via Alizarin Red staining, with expression of specific osteogenic markers determined via reverse transcription-quantitative PCR. It was found that crystalline GlcN sulfate was effective in decreasing osteoclastic cell differentiation and function. hOCs isolated from patients with OA were more sensitive compared with those from healthy donors. Additionally, GlcN exhibited anabolic effects on hOCs both in 2D conventional cell culture and in hOC/hOB 3D dynamic co-culture. The present study demonstrated for the first time the effectiveness of a 3D dynamic co-culture system for characterizing the spectrum of action
\end{abstract}

Correspondence to: Dr Letizia Penolazzi, Department of Neuroscience and Rehabilitation, University of Ferrara, Via Fossato di Mortara 74, I-44121 Ferrara, Italy

E-mail: pnlmlt@unife.it

Key words: glucosamine, bone tissue, bone cells, 3D culture system, osteoarthritis of GlcN on the bone microenvironment, which may pave the way for more fully determining the potential applications of a compound such as GlcN, which is positioned between pharmaceuticals and nutraceuticals. Based on the present findings, it is hypothesized that GlcN may have potential benefits in the treatment of osteopenic diseases such as osteoporosis, as well as in bone maintenance.

\section{Introduction}

Glucosamine $(\mathrm{GlcN})$ is an aminosaccharide that acts as a preferred substrate for the biosynthesis of glycosaminoglycans and, subsequently, for the production of aggrecan and other proteoglycans in the connective and cartilage tissues (1). GlcN supports joint structure function by serving as a building block of the cartilage matrix, and maintains joint health by preventing tissue degradation, reducing inflammation and oxidative stress, improving the autophagy response of chondrocytes and increasing the chondrogenic potential of mesenchymal stem cells resident in the niche $(1,2)$. Additionally, GlcN is an essential substrate for the synthesis of glycosylated proteins and lipids (3). For its biological properties, GlcN is prescribed as a drug or a dietary supplement in the management of one of the most common joint disorders, osteoarthritis (OA), to delay the progression of tissue degeneration and to attenuate the symptoms in humans $(1,4,5)$. Furthermore, GlcN is recommended for joint health to prevent sports-related cartilage injuries in athletes (6). At present, GlcN preparations are the most widely used nutraceutical for OA $(7,8)$. There are three common forms of GlcN supplements on the market: GlcN hydrochloride, GlcN sulfate, and N-acetyl GlcN. The chondroprotective action of these GlcN compound, is supported both by evidence obtained using different in vitro and in vivo experimental models, and also clinical trials (1). Currently, the prescription of crystalline GlcN sulfate (1,500 $\mathrm{mg}$ once daily) is recommended by the majority of clinical practice guidelines in the management of OA (9).

During the evolution of OA and disease progression, there are substantial subchondral bone metabolic alterations and remodeling (10); as OA in the elderly is often 
accompanied by osteoporosis (11), it is critical to also consider how bone tissue may be affected by GlcN. At present, there are limited data available concerning the effects of GlcN on human osteoclasts and osteoblasts that populate the bone microenvironment (12-14). It would be beneficial to obtain information concerning this in order to broaden the pharmacological relevance and potential therapeutic efficacy of GlcN in skeletal diseases.

The primary aim of the present pilot study was to examine the effects of GlcN on human primary osteoclasts (hOCs) cultured in conventional two-dimensional (2D) monolayer, as well as those in a more complex culture system that more closely models the in vivo bone microenvironment, consisting of an osteoclast/osteoblast 3D dynamic co-culture system (15). The employment of this in vitro model mimicking the process of bone matrix deposition and remodeling provides simultaneous information on osteoclast and osteoblast cell populations. The effects of crystalline GlcN sulfate on osteoclastogenesis were investigated, which was performed both by treatment with osteoclastogenic inducers or by the presence of osteoblasts. As a source of osteoclast progenitors, human primary monocytes (hMCs) from the peripheral blood of donors (healthy controls or patients with OA) were used.

\section{Materials and methods}

Reagents. DONA ${ }^{\circledR}$ (crystalline GlcN sulfate) was obtained from Mylan Italia S.r.l., resuspended at $50 \mathrm{mg} / \mathrm{ml}$ and stored at $4^{\circ} \mathrm{C}$. Histopaque ${ }^{\circledR}-1077$, ascorbic acid-2-phosphate, $\beta$-glycerophosphate, dexamethasone, MTT, Alizarin Red S (ARS), paraformaldehyde, Triton X-100, tartrate-resistant acid phosphatase (TRAP) kit (cat. no. 386), fetal calf serum (FCS), L-glutamine and antibiotics (penicillin and streptomycin) were purchased from Sigma-Aldrich (Merck KGaA). A High Capacity cDNA Reverse Transcription kit, TaqMan Gene Expression assays, Universal Master Mix II and Alexa Fluor $^{\circledR} 488$ Phalloidin (cat. no. A12379) were purchased from Thermo Fisher Scientific, Inc. Antibodies for human runt-related transcription factor 2 (Runx2; cat. no. sc-10758), collagen type $1 \alpha$ (COL1a1; cat. no. sc-28657), nuclear factor of activated T-cells, cytoplasmic 1 (NFATc1; cat. no. sc-13033) and cathepsin K (cat. no. sc-48353) were purchased from Santa Cruz Biotechnology, Inc., and osteopontin (OPN; clone LF-123) was a generous gift from Dr Larry Fisher (National Institutes of Health). High-glucose Dulbecco's modified Eagle's medium (DMEM), Ham's F12 and PBS were purchased from Euroclone SpA.

Cell isolation and culture. Female patients with OA $(n=7$; 50-74 years) and healthy volunteers $(n=4 ; 43-48$ years) were enrolled between May 2019 and December 2019 during routine medical check-ups at Centro di Medicina (Ferrara, Italy) after obtaining written informed consent; the study was approved by the Centro di Medicina's research committee (approval no. 172201). Briefly, peripheral blood mononuclear cells (PBMCs) were obtained from $20 \mathrm{ml}$ peripheral blood and separated using Histopaque-1077 as previously described (16). hMCs were purified from PBMCs via adhesion selection on polystyrene plates. PBMCs $\left(1 \times 10^{6} / \mathrm{cm}^{2}\right)$ were plated and allowed to settle for $4 \mathrm{~h}$ at $37^{\circ} \mathrm{C}$, and flasks were then rinsed to remove non-adherent cells. In order to confirm the ability of isolated hMCs to differentiate into mature osteoclasts (hOCs), macrophage colony-stimulating factor $(25 \mathrm{ng} / \mathrm{ml})$ and receptor activator of NF- $\kappa \mathrm{B}$ ligand (RANKL; $30 \mathrm{ng} / \mathrm{ml}$; PeproTech EC Ltd.) were added to the culture medium; after 14 days, TRAP staining was performed. The expression levels of the osteoclast-specific markers cathepsin K and NFATc1 were assessed via immunocytochemistry.

Human osteoblasts (hOBs) were obtained from vertebral laminae discarded during spinal surgery to remove lumbar herniated discs (Pfirrmann grade 2). Bone fragments were obtained between September 2019 and December 2019, after obtaining written informed consent from 4 donors with no comorbidity (43-48 years; 2 males and 2 females) using research protocols approved by the Ethics Committee of the University of Ferrara and St. Anna Hospital (approved on November 17, 2016). Briefly, bone fragments were placed in sterile PBS at $4^{\circ} \mathrm{C}$ and dissected within $16 \mathrm{~h}$ after removal. Bone chips were minced into smaller pieces as previously reported (17), washed twice with PBS, plated in T-25 culture flasks (Sarstedt, Inc.) and cultured in high-glucose DMEM/Ham's F12 (1:1) supplemented with 10\% FCS, 1 mM L-glutamine and antibiotics [(penicillin $(100 \mu \mathrm{g} / \mathrm{ml})$ and streptomycin, $(10 \mu \mathrm{g} / \mathrm{ml})]$. From each patient, a primary cell culture was obtained. Upon detection of a cell colony from the bone fragments (after 7 days), the cells were expanded until confluent [passage (P)0)]. The cells were then harvested after treatment with $0.05 \%$ trypsin EDTA for $2 \mathrm{~min}$ at $37^{\circ} \mathrm{C}$ (Sigma-Aldrich; Merck KGaA), washed, counted via hemocytometric analysis and used for further experiments (P1-3). During the culture period, cells were incubated at $37^{\circ} \mathrm{C}$ in a humidified atmosphere of $5 \% \mathrm{CO}_{2}$, and the medium was changed every 3 days. hOBs (P0) were characterized for the presence of OPN, Runx2 and COL1a1 via immunostaining.

Based on previous studies, osteogenic differentiation was performed by culturing hOBs for up to 14 days in osteogenic medium (OM) $(18,19)$ consisting of high-glucose DMEM, 10\% FCS, $10 \mathrm{mM} \beta$-glycerophosphate, $100 \mathrm{nM}$ dexamethasone and $100 \mu \mathrm{M}$ ascorbic acid-2-phosphate.

TRAP staining. TRAP staining of cells was performed as previously described (20). Briefly, the cells were fixed in 4\% PFA with 0.1 M cacodilic buffer, $\mathrm{pH} 7.2$ (0.1 M sodium cacodilate, $0.0025 \% \mathrm{CaCl}_{2}$ ) for $15 \mathrm{~min}$ at room temperature, extensively washed in the same buffer, and stained for TRAP according to the manufacturer's protocols. After washing with distilled water and drying, samples were observed under a Leica microscope (Leica Microsystems $\mathrm{GmbH}$ ). Mature TRAP-positive multinucleated cells containing $>3$ nuclei were counted as osteoclasts in 10 randomly selected optical fields for each sample (magnification, x20).

Immunocytochemistry. Immunocytochemical analysis was performed using an ImmPRESS Universal Reagent kit (Vector Laboratories, Inc.). hOCs or hOBs $\left(1 \times 10^{6} / \mathrm{cm}^{2}\right.$ and $1 \times 10^{4} / \mathrm{cm}^{2}$ cells, respectively) were seeded in $24-$-well plates, fixed in cold $100 \%$ methanol at room temperature for $10 \mathrm{~min}$ and permeabilized with $0.2 \%$ (v/v) Triton X-100 in TBS (1X). Then, the cells were treated in $0.3 \% \mathrm{H}_{2} \mathrm{O}_{2}$ in TBS (1X) for $10 \mathrm{~min}$ at room temperature, and subsequently incubated 
with ready-to-use $(2.5 \%)$ normal horse serum blocking solution (ImmPRESS Universal Reagent kit) for $15 \mathrm{~min}$ at room temperature.

After the incubation in blocking serum, cells were incubated at $4^{\circ} \mathrm{C}$ overnight following addition of the following rabbit anti-human polyclonal primary antibodies: Runx2 (1:200); COL1a1 (1:100); NFATc1 (1:300); cathepsin K (1:200); and OPN (1:200). After rinsing in $1 \mathrm{X}$ TBS, the cells were incubated for $30 \mathrm{~min}$ at room temperature with ImmPRESS reagent and then stained with substrate/chromogen mix (ImmPACT $\left.{ }^{\mathrm{TM}} \mathrm{DAB}\right)$. After washing, the cells were mounted in glycerol/PBS (9:1), counterstained with hematoxylin and observed with a Nikon Eclipse 50i optical microscope (magnification, x20; Nikon Corporation).

MTT assay. The effect of GlcN on hOC and hOB viability was assessed using MTT colorimetric assays. The cells were seeded in 96-well plates, treated with increasing concentrations of GlcN $(10,100$ and $200 \mu \mathrm{g} / \mathrm{ml})$ maintained at $37^{\circ} \mathrm{C}$. After $72 \mathrm{~h}$ of treatment, a solution of MTT in PBS was added to each well and the plate was incubated for $3 \mathrm{~h}$ at $37^{\circ} \mathrm{C}$. The MTT crystals were solubilized with $200 \mu 1$ lysis buffer (10\% SDS). Spectrophotometric absorbance of each sample was then measured at $570 \mathrm{~nm}$ by using a microplate reader (Sunrise $^{\mathrm{TM}}$ Absorbance Reader; Tecan Group, Ltd.). Live cells were calculated as a percentage of the control (untreated cells).

Apoptosis (TUNEL assay). At the end of osteoclastogenic induction, mature hOCs were treated with GlcN (100 and $200 \mu \mathrm{g} / \mathrm{ml}$ ) for $72 \mathrm{~h}$. The cells were then rinsed twice with PBS and fixed for $25 \mathrm{~min}$ in $4 \%$ PFA at room temperature. Apoptotic cells were detected using a DeadEnd Colorimetric Apoptosis Detection system (Promega Corporation) according to the manufacturer's instructions. Moreover, all cells were subjected to hematoxylin staining to reveal nuclei. The cells were mounted in glycerol/PBS (9:1) and observed under a Leica microscope (magnification, x20; Leica Microsystems $\mathrm{GmbH})$. The apoptotic rate was calculated as the percentage of apoptotic nuclei (dark brown nuclei) compared with the total number of nuclei of osteoclasts, evaluated in triplicate from each experimental sample (10 randomly selected optical fields/sample).

Phalloidin staining. For analysis of F-actin organization, hOCs were fixed with 4\% PFA for $10 \mathrm{~min}$ at room temperature, permeabilized with $0.1 \%$ Triton $\mathrm{X}-100$ for $15 \mathrm{~min}$ and stained with Alexa Fluor 488 Phalloidin (1:500 in PBS) for $30 \mathrm{~min}$ at room temperature. Nuclei were counterstained with DAPI for $2 \mathrm{~min}$ at room temperature. Fluorescent images were obtained using a fluorescence microscope, evaluated by two independent investigators in 10 randomly selected optical fields (magnification, x40; Nikon Eclipse 50i).

$R N A$ isolation and reverse transcription-quantitative $P C R$ (RT-qPCR). Total RNA was isolated from hOBs [2D culture in OM in the presence or absence of GlcN $(200 \mu \mathrm{g} / \mathrm{ml}$ )] by using an RNeasy Micro kit (Qiagen $\mathrm{GmbH}$ ) according to the manufacturer's instructions. RNA concentration and quality were measured using a NanoDrop $^{\mathrm{TM}}$ ND1000 UV-VIS spectrophotometer (Isogen
Life Science B.V.). cDNA was synthesized from total RNA in a $20 \mu \mathrm{l}$ reaction volume using a High Capacity cDNA RT kit, according to the manufacturer's instructions. Finally, $100 \mathrm{ng}$ cDNA was used for qPCR analysis. TaqMan Universal Master Mix II and probes for human alkaline phosphatase (ALP; assay no. Hs01029144_m1), Runx2 (assay no. Hs00231692_m1), OPN (assay no. Hs00959010_ m1), COL1A1 (assay no. Hs00164004_m1), osteocalcin (OCN; assay no. Hs01587813_g1), bone sialoprotein (BSP; assay no. Hs00913377_m1) were used according to the manufacturer's instructions. Thermocycling conditions for qPCR were as follows: Initial activation at $95^{\circ} \mathrm{C}$ for $10 \mathrm{~min}$, followed by 40 cycles of thermal denaturation at $95^{\circ} \mathrm{C}$ for $15 \mathrm{sec}$ and annealing/elongation at $60^{\circ} \mathrm{C}$ for $1 \mathrm{~min}$. RPL13a (assay no. Hs04194366_g1) was used for normalization of mRNA expression. Gene expression was assessed using a CFX96TM PCR detection system (Bio-Rad Laboratories, Inc.), and relative gene expression was calculated using the comparative $2^{-\Delta \Delta \mathrm{Cq}}$ method (21) and expressed as fold change. All reactions were performed in triplicate $(n=4)$.

hOBs/hOCs cultured in 3D dynamic system. The 3D dynamic culture conditions were set up using an RCCS-4 ${ }^{\mathrm{TM}}$ bioreactor (Synthecon, Inc.), with a High Aspect Ratio Vessel ${ }^{\mathrm{TM}}$ (HARV; Synthecon, Inc.). The HARV consists of a horizontally rotated culture chamber where the cells are suspended and a perfusion system with media continuously flowing through the culture chamber. The culture chamber can rotate in the X-axis at certain speeds (rpm); higher rpm values are associated with lower gravity. The rotation speed applied for the experiments was $4 \mathrm{rpm}$, corresponding to ground based dynamic culture in which aggregates are in continuous falling rotation close to the bottom of the vessel (3D-DycC conditions) (15).

hMCs from healthy donors were used as source of osteoclast progenitors and combined with hOBs from vertebral laminae to create a 3D culture system. Each aggregate was generated with unpooled cells from four different donors of hOBs and hMCs. hOB/hMC aggregates were generated in the absence of exogenous scaffolds. 3D-DycC dynamic co-culture conditions were applied as previously reported $(15,20)$. Briefly, $1-2 \times 10^{6} \mathrm{hOBs}$ and $0.5-1 \times 10^{6} \mathrm{hMCs}$ were inoculated into HARVs filled with high-glucose DMEM containing $10 \%$ FCS ( $2 \mathrm{ml})$; all air bubbles were removed from the culture chamber. Before treatment, the formation of spontaneously generated cell aggregates was verified at different cell ratios $(1: 1,1: 2,1: 3$ and conversely). The 2:1 hOBs/hMCs cell ratio was selected as the most effective condition to generate mature hOCs and applied for the following experiments.

HARV was then inserted into the RCCS-4 rotary bioreactor and placed in an incubator at $37^{\circ} \mathrm{C}$ with $5 \% \mathrm{CO}_{2}$. After $24 \mathrm{~h}$, the presence of aggregates was observed, and the vessels were filled with osteogenic medium alone $(\mathrm{OM})$ or in the presence of $200 \mu \mathrm{g} / \mathrm{ml} \mathrm{GlcN}(\mathrm{OM} / \mathrm{GlcN})$. Osteogenic medium and treatment with GlcN was refreshed twice a week. After 14 days, the aggregates were collected, fixed in 4\% PFA (15 min, room temperature) and embedded in paraffin for further analysis.

Histology. Immunohistochemistry was performed using the ImmPRESS Universal Reagent kit. Histological sections $(5 \mu \mathrm{m})$ of aggregates were subjected to 
A

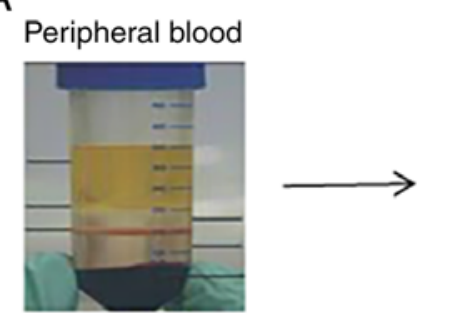

B

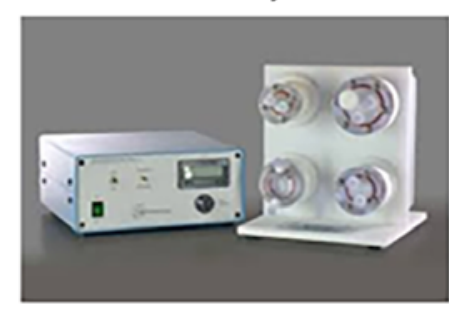

Osteoclasts

precursors (hMCs)

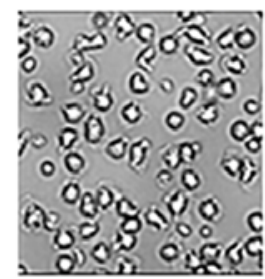

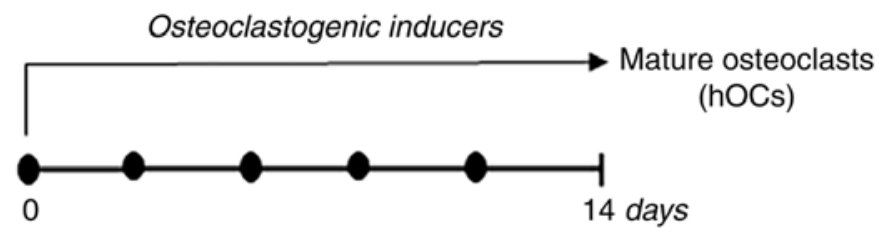

, + GICN

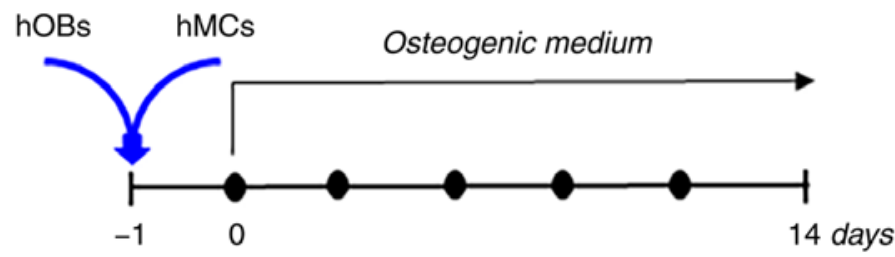

, + GlcN

Figure 1. Experimental set-up. (A) hOCs were obtained after culturing hMCs from the peripheral blood of healthy donors or patients with osteoarthritis for 14 days in osteoclastogenic medium. (B) hMCs were co-cultured with hOBs in a three-dimensional dynamic system to generate self-assembled aggregates in osteogenic medium. In both cases, cell cultures were exposed to GlcN, with medium renewed every 3 days. GlcN, glucosamine; hMC, human primary monocyte; hOB, human primary osteoblast; hOC, human primary osteoclast.

immunohistochemistry. Non-consecutive sections were deparaffinized, rehydrated and enzymatically treated with $1 \mathrm{mg} / \mathrm{ml}$ protease $\mathrm{K}$ for $10 \mathrm{~min}$ at $37^{\circ} \mathrm{C}$ (Sigma-Aldrich) for antigen retrieval and permeabilization. Slides were then immunostained overnight with primary antibodies against OPN (1:100) in a humid chamber at $4^{\circ} \mathrm{C}$, followed by treatment with ImmPRESS reagent (ImmPRESS reagent kit; Vector Laboratories, Inc.) for $30 \mathrm{~min}$. The reaction were developed using DAB solution (Vector Laboratories, Inc.); the sections were counterstained with hematoxylin, mounted in glycerol and observed using a Nikon Eclipse 50i optical microscope (magnification, $\mathrm{x} 10$ ).

For ARS staining, the sections were deparaffinized and stained with $40 \mathrm{mM}$ ARS solution ( $\mathrm{pH} 4.2)$ at room temperature for $20 \mathrm{~min}$. TRAP staining was conducted using the TRAP kit according to the manufacturer's protocols. Staining was quantified using a computerized video camera-based image analysis system ImageJ v1.51 software (http://rsb.info. nih.gov/nih-image/; National Institutes of Health) under light microscopy (magnification, x20; Nikon Eclipse 50i). Color TIFF file images were converted to 32-bit images and inverted so that the background could be set to the lower threshold limit. After applying the image threshold, the background was removed and not counted toward mean pixel intensity. Mean pixel intensity per area was used to quantify OPN staining (five sections/sample; $n=3$ ). The percentage positive area was used to quantify ARS and TRAP staining, accounting for tears/holes within the matrix of samples.

Statistical analysis. Results are presented as the mean \pm SD. Statistical significance was analyzed using GraphPad Prism 5 (GraphPad Software, Inc.) via one-way ANOVA followed by Tukey's post hoc test or Student's t-test. $\mathrm{P}<0.05$ was considered to indicate a significantly significant difference.

\section{Results}

GlcN induces apoptosis and decreases differentiation of osteoclasts from patients with OA. hMCs from peripheral blood of healthy controls or patients with OA were used as a source of osteoclast progenitors. The ability of hMCs to differentiate into mature multinucleated hOCs was demonstrated by analyzing the presence of established osteoclast markers, such as TRAP, cathepsin K and NFATc1, during osteoclastogenic induction. Exposure to different GlcN concentrations $(10-200 \mu \mathrm{g} / \mathrm{ml})$ did not affect osteoclast viability (Fig. S1A). Consistent with previous evidence $(12,22,23)$, it was selected to treat the cells with GlcN concentrations of 100 and $200 \mu \mathrm{g} / \mathrm{ml}$.

To investigate the effects of $\mathrm{GlcN}$ on hOCs, an experimental strategy was designed (Fig. 1), accounting for the low number of cells available from each patient sample that limited the experimental analysis that could be performed. Microscopic observations revealed that the number of multinuclear hOCs both from healthy donors or patients with OA was not significantly altered following GlcN treatment (Fig. S1B). After differentiation was completed, apoptosis was assessed using a TUNEL assay (Fig. 2). The results demonstrated that GlcN treatment induced dose-dependent cell apoptosis in hOCs from patients with OA, whereas hOCs from healthy donors underwent GlcN-induced DNA fragmentation only after exposure to $200 \mu \mathrm{g} / \mathrm{ml}$.

Considering that cytoskeletal rearrangements are a prerequisite for bone resorption by osteoclasts (24), the effects of $\mathrm{GlcN}$ on hOC differentiation were subsequently investigated by staining with FITC-conjugated phalloidin to evaluate actin ring formation. As shown in Fig. 3, GlcN treatment significantly decreased the polymerization of F-actin in a circular manner in hOCs from patients with OA, but not in hOCs from healthy donors. 

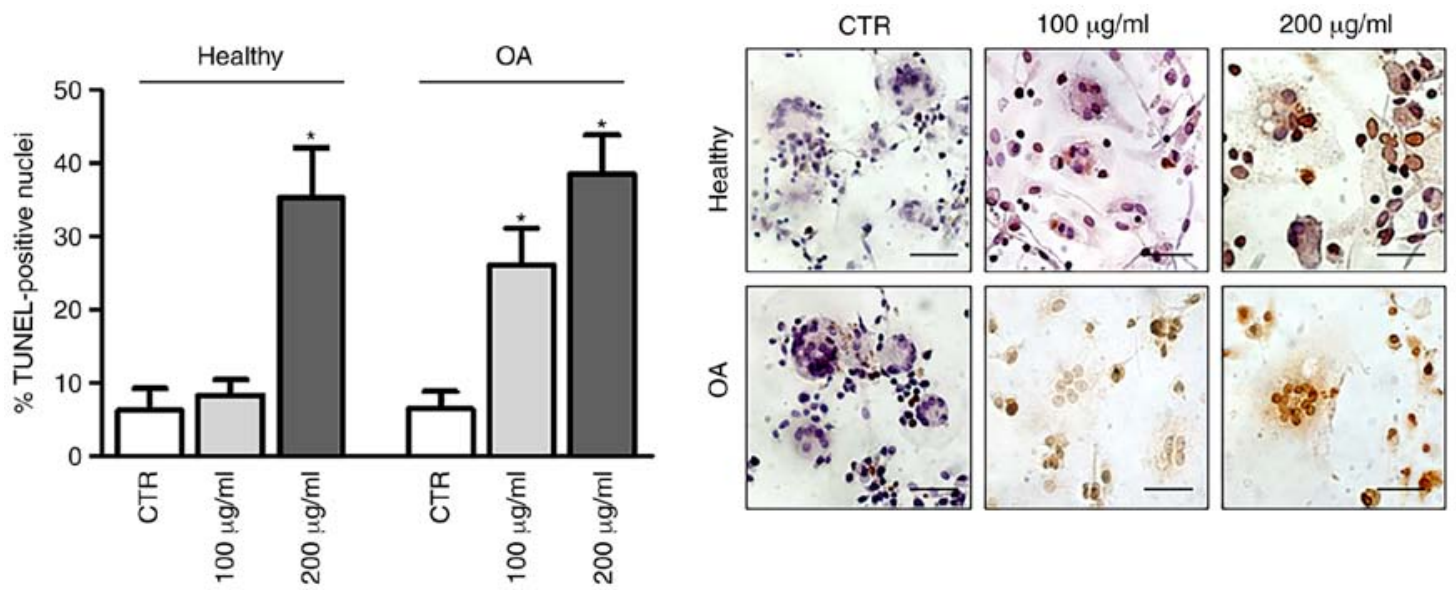

Figure 2. Effect of GlcN on hOC apoptosis. hOCs were incubated with 100 and $200 \mu \mathrm{g} / \mathrm{ml} \mathrm{GlcN}$ for $72 \mathrm{~h}$ and then subjected to TUNEL staining to detect apoptosis. hOCs were counterstained with hematoxylin. Scale bars, $50 \mu \mathrm{m}$. Data are presented as the percentage of TUNEL-positive nuclei (dark brown) when compared with the total number nuclei. Data are presented as the mean \pm SD. Healthy donors, $n=3$; patients with OA, $n=7$. ${ }^{*} \mathrm{P}<0.05$ vs. CTR. CTR, untreated control; GlcN, glucosamine; hOC, human primary osteoclast; OA, osteoarthritis.
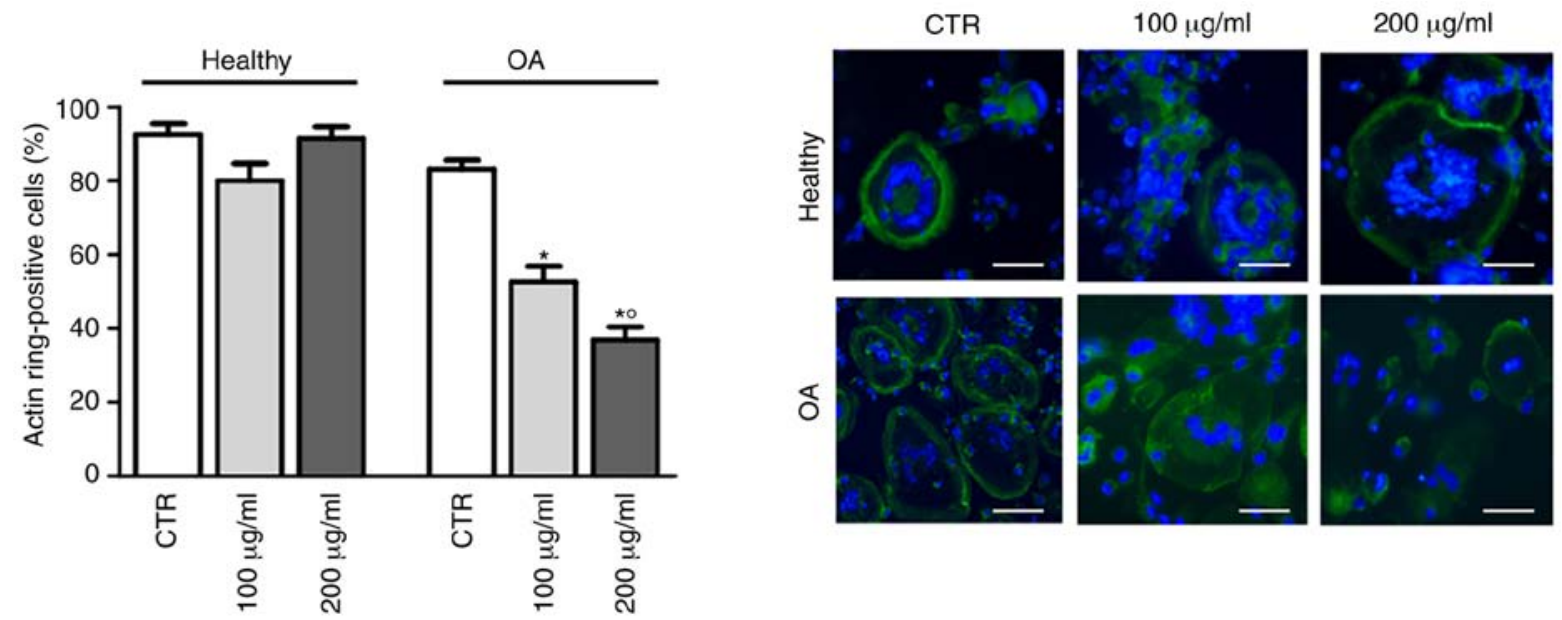

Figure 3. Effect of GlcN on hOC actin ring formation. Monocytes were cultured in osteoclastogenic medium in the absence or presence of GlcN (100 and $200 \mu \mathrm{g} / \mathrm{ml}$ ) for 14 days. hOC actin rings were analyzed via phalloidin staining; nuclei were counterstained with DAPI. Scale bars, $50 \mu \mathrm{m}$. Data are presented as the percentage of actin ring-positive cells relative to total number of cells, and were evaluated by two independent investigators in 10 randomly selected optical fields. Data are presented as the mean \pm SD. Healthy donors, $\mathrm{n}=3$; patients with $\mathrm{OA}, \mathrm{n}=7 .{ }^{*} \mathrm{P}<0.05 \mathrm{vs}$. CTR; ${ }^{\circ} \mathrm{P}<0.05 \mathrm{vs}$. $100 \mu \mathrm{g} / \mathrm{ml}$. CTR, untreated control; GlcN, glucosamine; hOC, human primary osteoclast; OA, osteoarthritis.

GlcN positively affects osteoblast activity in $h O C / h O B 3 D$ co-culture systems. Subsequent experiments investigated hOC responses to GlcN when combined with osteoblasts (hOBs) in a $3 \mathrm{D}$ co-culture system. The aim was to validate the hOC responsiveness to $\mathrm{GlcN}$ in an experimental condition that more closely resembles the in vivo bone microenvironment whilst also attempting to understand if hOBs could represent a GlcN target. The quality of the cells was assessed; only those hOB samples expressing conventional osteoblastic markers, such as OPN, COLla1 and Runx2 (Fig. S1C) were selected. When subjected to GlcN treatment up to $200 \mu \mathrm{g} / \mathrm{ml}$, hOBs did not exhibit any change in viability (Fig. S1C). Therefore, this concentration was selected for the subsequent experiments. hMC osteoclast precursors from healthy donors were then combined with hOBs in a $3 \mathrm{D}$ dynamic co-culture system in presence of $\mathrm{OM}$ without osteoclastogenic inducers, based on a previous protocol (20). Under these conditions, osteoclastogenesis was supported by hOBs and the cells were able to produce sizeable self-assembling aggregates (Fig. 4). After exposure to GlcN, it was observed that the relative TRAP-positive area significantly decreased (Fig. 4). Of note, GlcN exhibited a positive effect on osteoblast activity; a significant increase of both mineral matrix deposition (ARS-positive areas) and OPN expression was found in GlcN-treated cellular aggregates (Fig. 4).

Although some aspects of OPN function in bone homeostasis remain to be determined, migration, adhesion and activation of osteoclasts in an OPN-dependent manner have been demonstrated (25). However, it was hypothesized that the increase in OPN expression in the $\mathrm{hOC} / \mathrm{hOB} 3 \mathrm{D}$ co-culture system is to be attributed to the hOBs, as GlcN significantly increased ARS and decreased TRAP staining. Therefore, these results suggested that GlcN was effective not only in inhibiting the activity of hOBs, but also in enhancing the activity of hOCs.

This was further explored, as a number of osteogenic markers were analyzed via RT-qPCR after expanding 

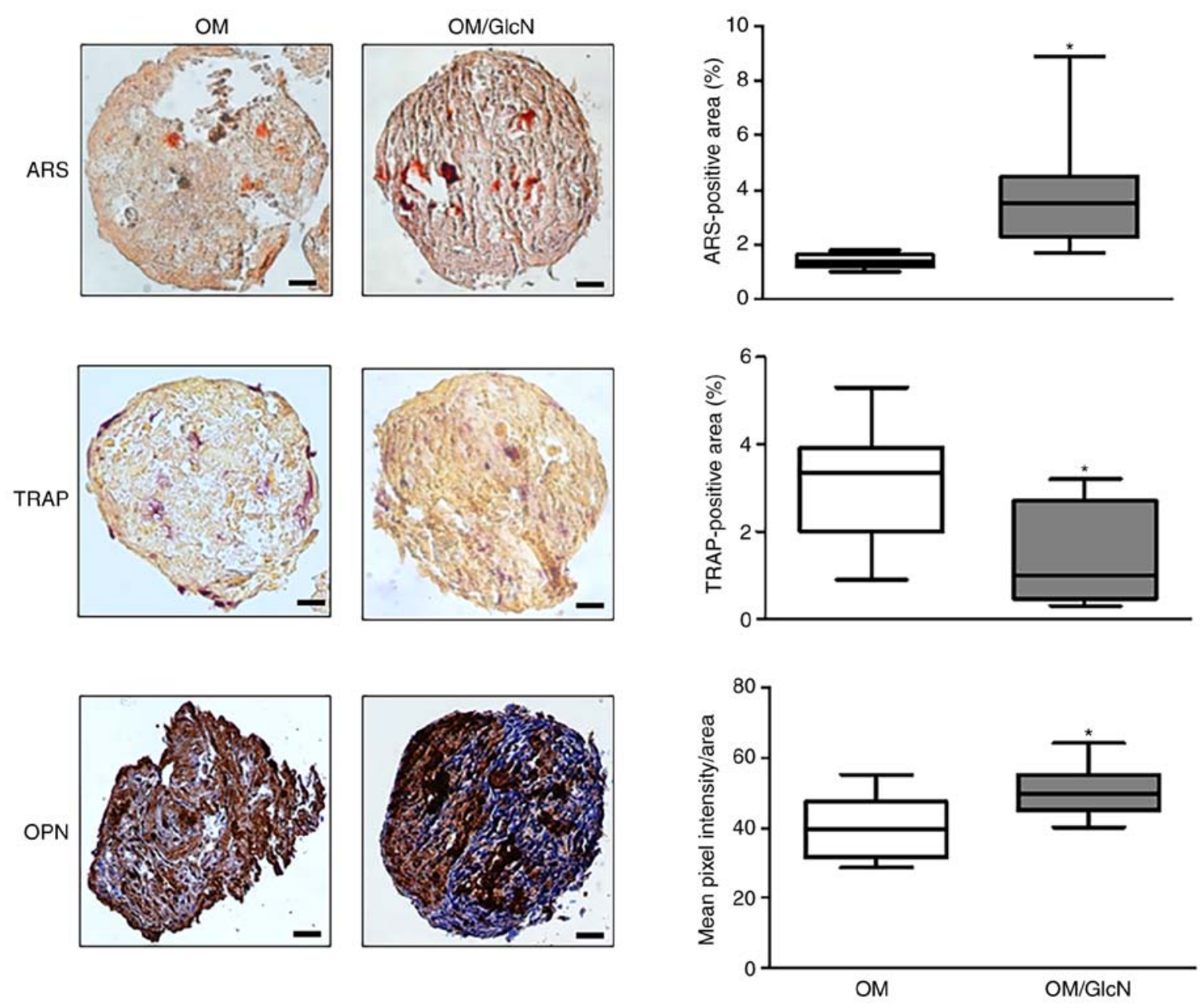

Figure 4. Responsiveness of hOCs and hOBs to GlcN in the 3D dynamic co-culture system. Human primary monocytes from healthy donors were co-cultured with hOBs for 14 days in a 3D dynamic system. Cells were cultured in OM or OM/GlcN. GlcN treatment was repeated every 3 days. Representative microphotographs of ARS, TRAP and OPN staining are reported. Scale bars, $50 \mu \mathrm{m}$. TRAP activity and ARS were quantified by ImageJ software and expressed as the percentage positive area (mean $\pm \mathrm{SD}$, five sections/sample, $\mathrm{n}=4$ ). OPN levels were quantified by ImageJ software and expressed as the mean pixel intensity/area (mean value $\pm \mathrm{SD}$, five sections/sample, $\mathrm{n}=3$ ). " $\mathrm{P}<0.05$ vs. OM. 3D, three-dimensional; ARS, Alizarin Red S; GlcN, glucosamine; hOC, human primary osteoclast; hOB, human primary osteoblast; OM, osteogenic medium; OM/GlcN, OM with $200 \mu \mathrm{g} / \mathrm{ml} \mathrm{GlcN}$; OPN, osteopontin; TRAP, tartrate-resistant acid phosphatase.

the hOBs in 2D conventional culture. As shown in Fig. 5, GlcN induced a general increase in early and middle stage osteogenic markers such as Runx2, COL1a1, ALP, OPN and BSP (26). In particular, a significant increase in expression was observed for Runx2, which is considered the master regulator of osteogenesis (26), and OPN. No significant changes in expression were observed for OCN, the late differentiation marker.

\section{Discussion}

Despite considerable knowledge of the biological activities of GlcN, including chondroprotective and anti-inflammatory actions (1-7), its role in osteogenesis and bone tissue remains to be investigated in detail. The evidence collected so far on bone cells is mainly based on the use of monolayered non-human cell lines, such as mouse MC3T3-E1 (14) or RAW264.7 (22), the fetal osteoblastic cell line hFOB1.19 (23), or animal models such as rats, mice or rabbits receiving GlcN oral administration (27-29).

The present study focused on cells from human bone microenvironments. Initial experiments involved peripheral blood samples from patients with OA; as these patients were outpatients who did not require surgery, it was only possible to obtain a limited amount of peripheral blood, although this was sufficient to produce the hOC precursors for a comparative study with hOCs from healthy donors. This may be a limitation of this study; in the near future, there are plans to enroll patients with OA that require orthopedic surgery, so that both endogenous osteoclasts and osteoblasts can be obtained to conduct a greater number of analysis. Nevertheless, the present data demonstrated that OA and healthy osteoclasts were differentially susceptible to GlcN treatment, which inhibited the differentiation and function of OA osteoclasts.

These findings led to subsequent investigations into the effects of GlcN in a more complex culture system one step closer to the in vivo bone microenvironment, consisting of an hOC/hOB 3D dynamic co-culture system. With this approach, the effect of GlcN on osteoclast behavior was validated, revealing a decrease in TRAP activity, but the responsiveness of human osteoblasts was also investigated. After GlcN treatment, osteoblasts increased mineral matrix deposition and the expression of specific differentiation markers, such 

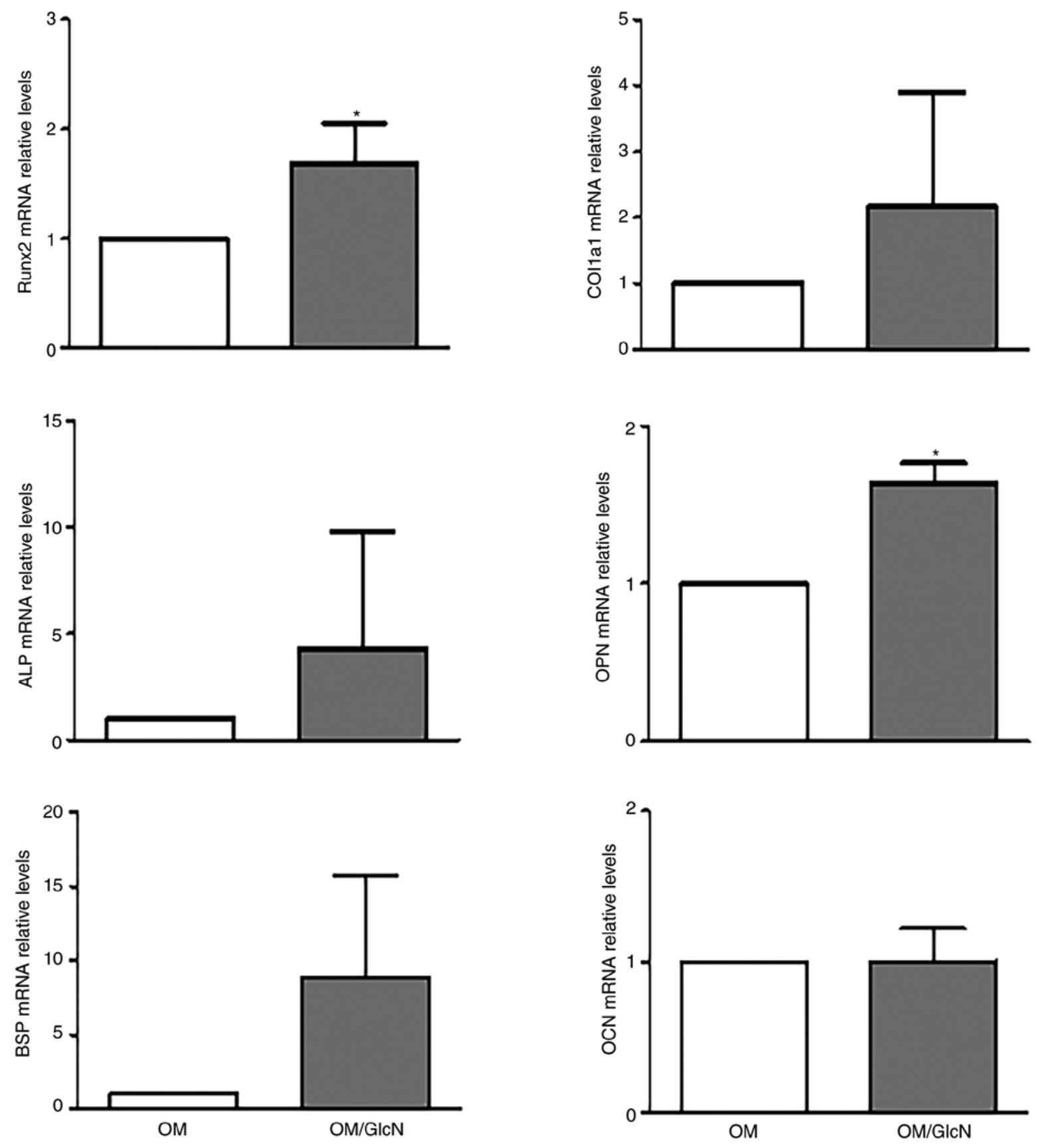

Figure 5. Effect of GlcN on hOBs in two-dimensional conventional cell culture. The expression of typical osteogenic markers was analyzed in hOBs cultured in $\mathrm{OM}$ or $\mathrm{OM} / \mathrm{GlcN}$ for 14 days. GlcN treatment was repeated every 3 days. Total RNA was purified, and the mRNA expression levels of Runx2, COL1a1, ALP, OPN, BSP and OCN were evaluated via reverse transcription-quantitative PCR. Relative expression levels were normalized to OM. All reactions were performed in triplicate. Data are presented as the mean $\pm \mathrm{SD}(\mathrm{n}=4)$. ${ }^{*} \mathrm{P}<0.05$ vs. OM. ALP, alkaline phosphatase; BSP, bone sialoprotein; COL1a1, collagen type $1 \alpha$; GlcN, glucosamine; hOB, human primary osteoblast; OCN, osteocalcin; OM, osteogenic medium; OM/GlcN, OM with $200 \mu \mathrm{g} / \mathrm{ml}$ GlcN; OPN, osteopontin; Runx2, runt-related transcription factor 2 .

as OPN, demonstrating the ability of GlcN to exert anabolic effects. This is an encouraging proof of concept that needs to be validated in the future through the use of a larger number of cells, which will allow for analysis of a larger number of osteogenic markers. The reduced amount of cells harvestable from patients and issues during the aggregate post-culturing process had narrowed the number of experimental analyses performed.

When GlcN treatment was performed on 2D conventional osteoblast culture and RT-qPCR analysis of differentiation markers was conducted, the aforementioned findings were validated, demonstrating that GlcN supported favorable conditions for osteogenic differentiation and maintenance of osteoblastic phenotypes.

The opposing responses of the different bone cell populations merits further study; however, the present findings suggest that GlcN may be a candidate as a broader treatment/therapeutic aimed at resolving both cartilage and skeletal diseases. Additionally, it is proposed that the results of research conducted in this area will help clinicians with providing a broader and more targeted prescription of GlcN, whilst providing benefits to patients and their bone tissues. 
It is important to underline that identifying molecules capable of simultaneously modulating the activity of osteoblasts and osteoclasts is an important benefit for patients affected by bone loss, as it provides the opportunity to control a complex balance $(30,31)$. It is well known that bone deposition by osteoblasts and resorption by osteoclasts are tightly coupled, and their balance defines both the mass and quality of bone tissue (30). Using culture conditions to the in vivo bone microenvironment such as those reported in the present study provides a novel perspective, both by generating informative data on the still-controversial efficacy of biological agents such as GlcN and by conducting patient-oriented research. This last aspect is based on the possibility of generating autologous osteoclast/osteoblast 3D co-cultures with cells from the same patient, who, in addition to peripheral blood, may provide bone fragments during orthopedic surgery. Therefore, the employment of such an approach may further improve understanding of the role of GlcN in bone tissue homeostasis, as well as the development of patient-tailored nutraceutical and pharmaceutical treatments $(32,33)$.

The effects of GlcN reported in the present study are consistent with the only other study, to the authors' knowledge, into the human bone microenvironment, namely that by Tat et al (12). In this paper, the authors studied the effect of chondroitin sulfate, GlcN sulfate and vitamin D3 on osteoblast metabolism in the subchondral bone of patients with OA, demonstrating that GlcN decreased osteoblast pro-resorptive activity by modulating osteoprotegerin/RANKL signaling (12).

At present, understanding how the altered bone remodeling that supports the development of both osteoarthritis and osteoporosis can be counteracted by adequate $\mathrm{GlcN}$ treatment in terms of dose and intake remains an open question. For this reason, clinical studies have to be accompanied by the development of suitable preclinical experimental models that provide useful information on exact mechanisms of action underlying the beneficial effects of GlcN.

It is worth mentioning that, in addition to the well-known role of GlcN in the synthesis of components of the extracellular matrix $(1,34)$, it is the precursor of $\mathrm{N}$-acetyl-GlcN, which is added to the serine and threonine residues of nuclear and cytoplasmic proteins in the O-GlcNAcylation post-translational modification (35). O-GlcNAcylation plays a critical role in the regulation of cellular homeostasis in response to nutritional or hormonal cues, and also in response to stress or damage (36). A previous study reported that an increase of global O-GlcNAc glycosylation occurs during the early stages of osteoblast differentiation in MC3T3-E1 cells, but not during the osteoclastic differentiation of RAW264 cells (37). Considering that acute and chronic alterations in the amount of O-GlcNAcylated proteins have been associated with different human diseases (38), a key point that requires further investigation will be to clarify the involvement of O-GlcNAc glycosylation in altered bone metabolism and the modulation of osteogenic gene expression in human bone cells.

Collectively, the present findings provided evidence that compounds such as GlcN that are positioned between pharmaceuticals and nutraceuticals merit further investigation for developing novel approaches for bone health maintenance and treatment of bone diseases.

\section{Acknowledgements}

We wish to thank Dr Francesco Nicoli (Department of Chemical and Pharmaceutical Sciences-University of Ferrara-Italy) and Dr Leticia Scussel Bergamin (Department of Neuroscience and Rehabilitation, University of Ferrara-Italy) for technical assistance, and Professor Pasquale De Bonis (Department of Translational Medicine and for Romagna-University of Ferrara-Italy) for providing bone surgical fragments.

\section{Funding}

This work was supported by the CCIAA 2018 -UniFE fund (grant no. 167349).

\section{Availability of data and materials}

The datasets used and/or analyzed during the current study are available from the corresponding author on reasonable request.

\section{Authors' contributions}

LP designed the study, performed the experiments and analyzed the data. EL designed the study, performed the experiments and analyzed the data. AP analyzed the data and reviewed the manuscript. DM analyzed the data and reviewed the manuscript. VS designed and coordinated the study, and helped with the interpretation of data. RP designed the study, and wrote and edited the manuscript. All authors read and approved the final manuscript.

\section{Ethics approval and consent to participate}

Approval for the study was obtained from the Centro di Medicina (Ferrara, Italy) and from the Ethics Committee of the University of Ferrara and St. Anna Hospital (protocol approved on November 17, 2016). Written informed consent was obtained from each patient. No animals were involved in the present study.

\section{Patient consent for publication}

Not applicable.

\section{Competing interests}

The authors declare that they have no competing interests.

\section{References}

1. Nagaoka I, Igarashi M and Sakamoto K: Biological activities of glucosamine and its related substances. Adv Food Nutr Res 65: 337-352, 2012

2. Varghese S, Theprungsirikul P, Sahani S, Hwang N, Yarema KJ and Elisseeff JH: Glucosamine modulates chondrocyte proliferation, matrix synthesis, and gene expression. Osteoarthritis Cartilage 15: 59-68, 2007.

3. Reily C, Stewart TJ, Renfrow MB and Novak J: Glycosylation in health and disease. Nat Rev Nephrol 15: 346-366, 2019.

4. Block JA, Oegema TR, Sandy JD and Plaas A: The effects of oral glucosamine on joint health: Is a change in research approach needed? Osteoarthritis Cartilage 18: 5-11, 2010. 
5. Agiba AM: Nutraceutical formulations containing glucosamine and chondroitin sulphate in the treatment of osteoarthritis: Emphasis on clinical efficacy and formulation challenges. Int J Curr Pharm Res 9: 1-7, 2017.

6. Ostojic SM, Arsic M, Prodanovic S, Vukovic J and Zlatanovic M: Glucosamine administration in athletes: Effects on recovery of acute knee injury. Res Sports Med 15: 113-124, 2007.

7. D'Adamo S, Cetrullo S, Panichi V, Mariani E, Flamigni F and Borzì RM: Nutraceutical activity in osteoarthritis biology: A focus on the nutrigenomic role. Cells 9: 1232, 2020.

8. Ragle RM and Sawitzke AD: Nutraceuticals in the management of osteoarthritis: A critical review. Drugs Aging 29: 717-731, 2012

9. Rovati LC, Girolami F and Persiani S: Crystalline glucosamine sulfate in the management of knee osteoarthritis: Efficacy, safety, and pharmacokinetic properties. Ther Adv Musculoskelet Dis 4: 167-180, 2012

10. Goldring SR and Goldring MB: Changes in the osteochondral unit during osteoarthritis: Structure, function and cartilage-bone crosstalk. Nat Rev Rheumatol 12: 632-644, 2016.

11. Im GI and Kim MK: The relationship between osteoarthritis and osteoporosis. J Bone Miner Metab 32: 101-109, 2014.

12. Tat SK, Pelletier JP, Vergés J, Lajeunesse D, Montell E, Fahmi H, Lavigne $\mathrm{M}$ and Martel-Pelletier J: Chondroitin and glucosamine sulfate in combination decrease the pro-resorptive properties of human osteoarthritis subchondral bone osteoblasts: A basic science study. Arthritis Res. Ther 9: R117, 2007.

13. Anastassiades T, Rees-Milton K, Xiao H, Yang X, Willett T and Grynpas M: N-acylated glucosamines for bone and joint disorders: Effects of N-butyryl glucosamine on ovariectomized rat bone. Transl Res 162: 93-101, 2013.

14. Igarashi M, Sakamoto K and Nagaoka I: Effect of glucosamine, a therapeutic agent for osteoarthritis, on osteoblastic cell differentiation. Int J Mol Med 28: 373-379, 2011.

15. Penolazzi L, Lolli A, Sardelli L, Angelozzi M, Lambertini E, Trombelli L, Ciarpella F, Vecchiatini R and Piva R: Establishment of a 3D-dynamic osteoblasts-osteoclasts co-culture model to simulate the jawbone microenvironment in vitro. Life Sci 152: 82-93, 2016.

16. Penolazzi L, Pocaterra B, Tavanti E, Lambertini E, Vesce F, Gambari R and Piva R: Human osteoclasts differentiated from umbilical cord blood precursors are less prone to apoptotic stimuli than osteoclasts from peripheral blood. Apoptosis 13: 553-561, 2008.

17. Lambertini E, Penolazzi L, Angelozzi M, Grassi F, Gambari L, Lisignoli G, De Bonis P, Cavallo M and Piva R: The expression of cystathionine gamma-lyase is regulated by estrogen receptor alpha in human osteoblasts. Oncotarget 8: 101686-101696, 2017.

18. Wrobel E, Leszczynska J and Brzoska E: The characteristics of human bone-derived cells (HBDCS) during osteogenesis in vitro. Cell Mol Biol Lett 21: 26, 2016.

19. Choudhary S, Sun Q, Mannion C, Kissin Y, Zilberberg J and Lee WY: Hypoxic three-dimensional cellular network construction replicates ex vivo the phenotype of primary human osteocytes. Tissue Eng Part A 24: 458-468, 2018.

20. Mandatori D, Penolazzi L, Pipino C, Di Tomo P, Di Silvestre S, Di Pietro N, Trevisani S, Angelozzi M, Ucci M, Piva R and Pandolfi A: Menaquinone-4 enhances osteogenic potential of human amniotic fluid mesenchymal stem cells cultured in 2D and 3D dynamic culture systems. J Tissue Eng Regen Med 12: 447-459, 2018.

21. Livak KJ and Schmittgen TD: Analysis of relative gene expression data using real-time quantitative PCR and the 2(-Delta Delta C(T)) method. Methods 25: 402-408, 2001
22. Takeuchi T, Sugimoto A, Imazato N, Tamura M, Nakatani S, Kobata K and Arata Y: Glucosamine suppresses osteoclast differentiation through the modulation of glycosylation including O-GlcNAcylation. Biol Pharm Bull 40: 352-356, 2017.

23. Lv C, Wang L, Zhu X, Lin W, Chen X, Huang Z, Huang L and Yang S: Glucosamine promotes osteoblast proliferation by modulating autophagy via the mammalian target of rapamycin pathway. Biomed Pharmacother 99: 271-277, 2018.

24. Matsubara T, Kinbara M, Maeda T, Yoshizawa M, Kokabu S and Yamamoto TT: Regulation of osteoclast differentiation and actin ring formation by the cytolinker protein plectin. Biochem Biophys. Res Commun 489: 472-476, 2017.

25. Luukkonen J, Hilli M, Nakamura M, Ritamo I, Valmu L, Kauppinen K, Tuukkanen J and Lehenkari P: Osteoclasts secrete osteopontin into resorption lacunae during bone resorption. Histochem Cell Biol 151: 475-487, 2019.

26. Chapurlat RD and Confavreux CB: Novel biological markers of bone: From bone metabolism to bone physiology. Rheumatology (Oxford) 55: 1714-1725, 2016.

27. Jiang Z, Li Z, Zhang W, Yang Y, Han B, Liu W and Peng Y: Dietary natural N-Acetyl-d-Glucosamine prevents bone loss in ovariectomized rat model of postmenopausal osteoporosis. Molecules 23: 2302, 2018

28. Ivanovska $\mathrm{N}$ and Dimitrova P: Bone resorption and remodeling in murine collagenase-induced osteoarthritis after administration of glucosamine. Arthritis Res Ther 13: R44, 2011.

29. Wang SX, Laverty S, Dumitriu M, Plaas A and Grynpas MD: The effects of glucosamine hydrochloride on subchondral bone changes in an animal model of osteoarthritis. Arthritis Rheum 56: 1537-1548, 2007.

30. Feng $X$ and McDonald JM: Disorders of bone remodeling. Annu Rev Pathol 6: 121-145, 2011.

31. Kim BJ and Koh JM: Coupling factors involved in preserving bone balance. Cell Mol Life Sci 76: 1243-1253. 2019.

32. Nasri H, Baradaran A, Shirzad H and Rafieian-Kopaei M: New concepts in nutraceuticals as alternative for pharmaceuticals. Int J Prev Med 5: 1487-1499, 2014.

33. Daliu P, Santini A and Novellino E: From pharmaceuticals to nutraceuticals: Bridging disease prevention and management. Expert Rev Clin Pharmacol 12: 1-7, 2019.

34. Felson DT: Concerns about report suggesting glucosamine and chondroitin protect against cartilage loss. Ann Rheum Dis 74: e38, 2015.

35. Yang X and Qian K: Protein O-GlcNAcylation: Emerging mechanisms and functions. Nat Rev Mol Cell Biol 18: 452-465, 2017.

36. Herrero-Beaumont $G$ and Largo R: Glucosamine and O-GlcNAcylation: A novel immunometabolic therapeutic target for OA and chronic, low-grade systemic inflammation? Ann Rheum Dis 79: 1261-1263, 2020.

37. Koyama $\mathrm{T}$ and Kamemura K: Global increase in O-linked $\mathrm{N}$-acetylglucosamine modification promotes osteoblast differentiation. Exp Cell Res 338: 194-202, 2015.

38. Hart GW, Slawson C, Ramirez-Correa G and Lagerlof O: Cross talk between O-GlcNAcylation and phosphorylation: Roles in signaling, transcription, and chronic disease. Annu Rev Biochem 80: 825-858, 2011.

This work is licensed under a Creative Commons Attribution-NonCommercial-NoDerivatives 4.0 International (CC BY-NC-ND 4.0) License. 